\title{
Prediction of Bacillus Calmette-Guerin Response in Patients with Bladder Cancer after Transurethral Resection of Bladder Tumor by Using Genetic Variation Based on Genomic Studies
}

\author{
Ning Zhang, ${ }^{1,2,3}$ Guangliang Jiang, ${ }^{1,2}$ Xu Liu, ${ }^{3,4}$ Rong Na, ${ }^{1,2,3}$ \\ Xiang Wang, ${ }^{5}$ and Jianfeng $\mathrm{Xu}^{2,3}$ \\ ${ }^{1}$ Department of Urology, Huashan Hospital, Fudan University, Shanghai, China \\ ${ }^{2}$ Fudan Institute of Urology, Huashan Hospital, Fudan University, Shanghai, China \\ ${ }^{3}$ Program for Personalized Cancer Care, NorthShore University HealthSystem, Chicago, IL 60201, USA \\ ${ }^{4}$ Fudan Center for Genetic Epidemiology, School of Life Sciences, Fudan University, Shanghai, China \\ ${ }^{5}$ Department of Urology, Shanghai First People's Hospital, School of Medicine, Shanghai Jiao Tong University, Shanghai, China
}

Correspondence should be addressed to Rong Na; narong.hs@gmail.com and Xiang Wang; seanw_hs@163.com

Received 8 August 2016; Revised 18 October 2016; Accepted 31 October 2016

Academic Editor: Hushan Yang

Copyright (C) 2016 Ning Zhang et al. This is an open access article distributed under the Creative Commons Attribution License, which permits unrestricted use, distribution, and reproduction in any medium, provided the original work is properly cited.

\begin{abstract}
Purpose. We aimed to comprehensively review contemporary literature on genetic and epigenetic biomarkers associated with the prediction of Bacillus Calmette-Guerin (BCG) response after the transurethral resection of a bladder tumor and to discuss the application of these biomarkers in precision cancer care for bladder cancer. Method. We performed a systematic review of published literatures in the databases PubMed and Embase by using the following key words: bladder cancer, BCG, gene, and methylation. Studies associated with cell lines, animal models, and muscle invasive bladder cancer were excluded. Results. The genetic variations associated with BCG response can be classified into three categories: germline variations, somatic variations, and epigenetic alterations. Genes related to BCG response were mainly involved in single-nucleotide polymorphisms, copy number variations, and gene methylations. Conclusions. Although these gene alterations are currently the most promising predictive markers of BCG response, most studies about bladder cancer DNA biomarkers are related to germline variations in candidate genes, and the results are not consistent. Only one study is related to somatic variation, and further evaluation in large-scale validation studies should be conducted to assess the potential clinical application of these findings. In addition, other biomarkers based on different "-omics" technologies should be considered in future studies.
\end{abstract}

\section{Background}

Bladder cancer (BC) is the most common malignancy of the urinary tract and the 7th most common cancer in men and the 17 th in women [1]. Nearly $75 \%$ of bladder cancer patients are nonmuscle invasive bladder cancer (NMIBC), which can be treated with transurethral resection of bladder tumor (TURBT). Postoperative intravesical treatments are recommended based on clinical guidelines. For patients with intermediate-risk and high-risk tumors, intravesical fulldose Bacillus Calmette-Guerin (BCG) instillation is recommended as the first choice of treatment [2]. However, nearly $40 \%$ of patients do not respond to intravesical BCG therapy and ultimately experience disease recurrence or progression, which leads to a poorer prognosis $[3,4]$. Due to the limitations of one-size-fits-all clinical treatment of bladder cancer using BCG, it is of clinical importance to predict individual response to BCG treatment and provide personalized treatment for these patients. For example, using mitomycin or other chemotherapy agents for bladder instillation in patients who do not response to BCG may improve the disease prognosis.

Genetic alterations have been reported to play important roles in BCG response. First, genetic alterations in single nucleotides in numerous pathways were found to be closely associated with BCG response. These alterations included 


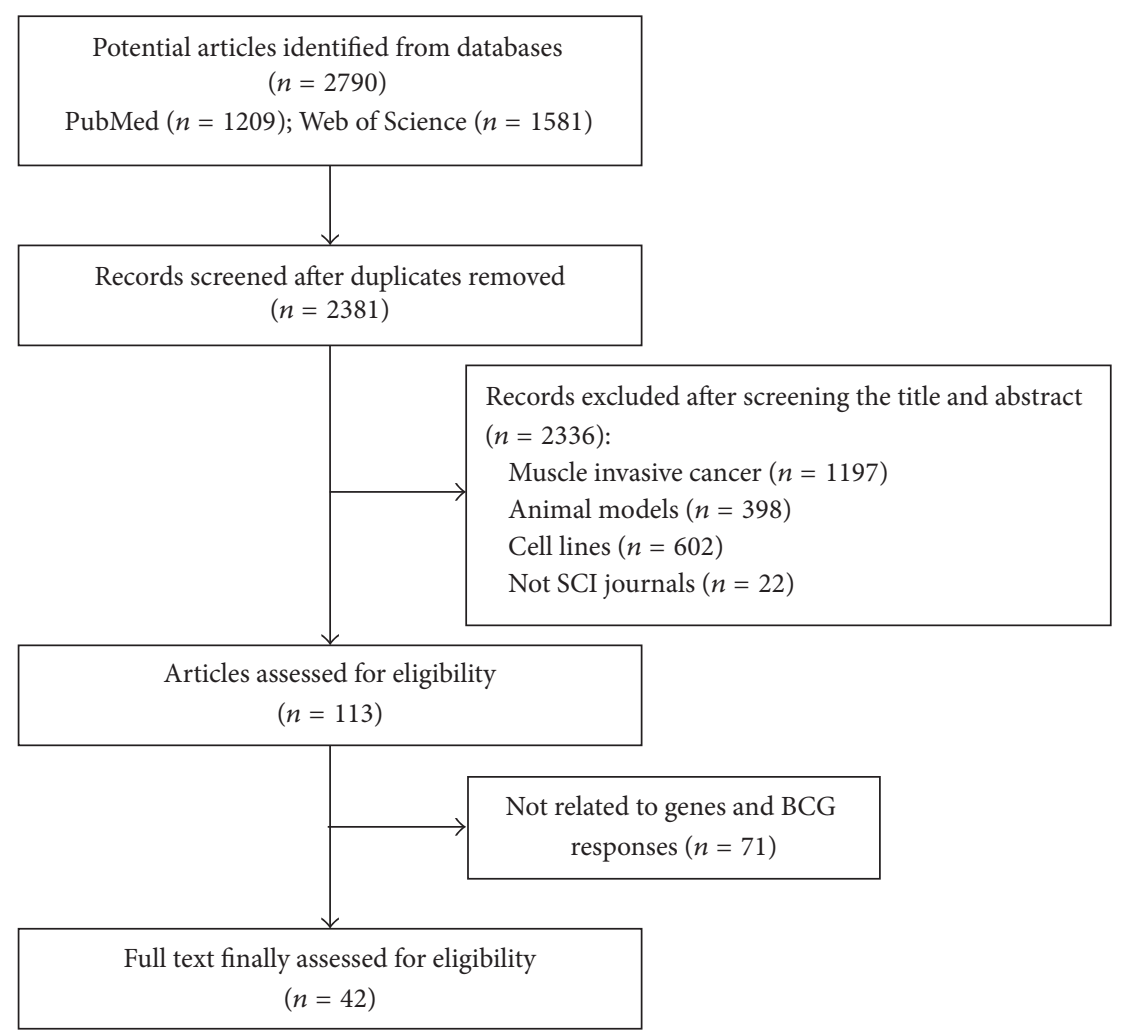

FIGURE 1: Flowchart of study selection process.

single-nucleotide polymorphisms (SNPs), single-nucleotide variations (SNVs), and mutations $[5,6]$. Second, various genetic copy number alterations were associated with bladder cancer [7]. Third, epigenetic regulation of genes, especially by gene methylation, was found to be associated with BCG failure for multiple genes [8-10].

There have been few systematic review papers associated with BCG response in the past 15 years and only one systematic review paper published in 2012 [11]. In this systematic review, we aimed to review the contemporary literature on biomarkers predicting BCG response, particularly in genetics and epigenetics.

\section{Evidence Acquisition}

A systematic review of published literature based on population studies published during 2000-2016 in PubMed and Embase databases was performed. The following keywords were used: bladder cancer, BCG, gene, SNP, copy number, and methylation. Studies involved in cell lines, animal models, and muscle invasive bladder cancer were excluded. Published articles that did not belong to the science citation index were also excluded. Ultimately, 2790 articles were found based on the keywords, 42 articles of which that had full text available were reviewed in detail, and 22 of them were included based on exclusion criteria (Figure 1). All articles were examined by two authors, and papers related to our research purpose were selected.

\section{Evidence Synthesis}

\subsection{Germline Variations}

3.1.1. Inflammatory Genes. The antitumor activities of BCG were related closely to immune system activation, so cytokines and immune cells played the key role in the process of antitumor. Therefore, polymorphisms of genes involved in the processes of BCG immunotherapy mechanism would affect the BCG response [12].

Single-nucleotide polymorphisms (SNPs) within the interleukin family were found to be associated with BCG response (Table 1). Leibovici et al. found that SNP-174(C/C) at $I L-6$ was associated with an increased risk of disease recurrence (HR: 4.60) in patients receiving BCG after TURBT [13]. However, another study indicated that SNP-174(C/C) at $I L-6$ was associated with a decreased risk of recurrence $(\mathrm{HR}=0.298)$ and an increased recurrence-free survival (RFS) [14]. Studies on TNF- $a$ polymorphisms reported that the SNP rs1799964(C/C) at TNF- $a$ was associated with a decreased risk of recurrence $[6,15]$. Ahirwar et al. found that rs 4073 (at $I L-8)$ was significantly associated with a decreased risk of recurrence $(p<0.001)$ and an increased RFS $(p<0.001)$ in patients who underwent BCG treatment after TURBT, but these results have not yet been confirmed [13]. Rs2070874 at $I L-4$, rs 2104286 at IL2RA, and rs4819554 at IL17RA were also reported to be associated with disease recurrence after BCG treatment [6]. 
TABLE 1: Inflammatory genes.

\begin{tabular}{|c|c|c|c|}
\hline Study & Sample (number) & Genes & Description \\
\hline Leibovici et al. [13] & 135 & $I L-6(-174 \mathrm{C} / \mathrm{C})$ & $\begin{array}{c}\text { It was associated with a } \\
\text { decreased recurrence risk }(\mathrm{HR}= \\
0.298 ; p=0.03) \text { and an increased } \\
\text { RFS }(p=0.021) \text { after BCG }\end{array}$ \\
\hline Ahirwar et al. [14] & 69 & $I L-6(-174 \mathrm{C} / \mathrm{C})$ & $\begin{array}{l}\text { It was associated with an } \\
\text { increased recurrence risk (HR: } \\
4.60 \text { ) in patients receiving BCG }\end{array}$ \\
\hline Lima et al. [6] & 204 & TNF- $a(\mathrm{rs} 1799964 \mathrm{C} / \mathrm{C})$ & Both of them were \\
\hline Ahirwar et al. [15] & 73 & TNF- $a(\mathrm{rs} 1799964 \mathrm{C} / \mathrm{C})$ & $\begin{array}{l}\text { associated with a decreased } \\
\text { recurrence risk }\end{array}$ \\
\hline Ahirwar et al. [17] & 71 & $I L-8(\mathrm{rs} 4073 \mathrm{~A} / \mathrm{A})$ & $\begin{array}{l}\text { It was associated with a decreased } \\
\text { recurrence risk }(p<0.001) \text { and } \\
\text { an increased RFS }(p<0.001)\end{array}$ \\
\hline Leibovici et al. [13] & 135 & $I L-8(\mathrm{rs} 4073 \mathrm{~A} / \mathrm{A})$ & There was no association \\
\hline Lima et al. [6] & 204 & $\begin{array}{c}\text { IL4(rs2070874 C/C) } \\
\text { TRAILR1(rs79037040 T/T) } \\
\text { IL2RA(rs2104286 T/T) } \\
\text { IL17RA(rs4819554 A/A })\end{array}$ & $\begin{array}{l}\text { They were associated with an } \\
\text { elevated risk of recurrence }\end{array}$ \\
\hline Ahirwar et al. [17] & 71 & $N F-k B(-94$ ATTGdel/del) & \\
\hline Ahirwar et al. [16] & 73 & $\begin{array}{c}I F N-G(+874 \mathrm{~A} / \mathrm{A}) \\
T G F-B(+28 \mathrm{~T} / \mathrm{T})\end{array}$ & $\begin{array}{l}\text { It demonstrated protective } \\
\text { association }\end{array}$ \\
\hline
\end{tabular}

SNPs at other immune factor genes were also found to be related to BCG response. Studies showed that TGF$B(+28 \mathrm{~T} / \mathrm{T})$ was associated with a decreased risk of recurrence [16], while $\mathrm{rs} 79037040(\mathrm{~T} / \mathrm{T})$ at TRAILR1 and IFN$G(+874 \mathrm{~A} / \mathrm{A})$ and $\mathrm{rs} 2104286(\mathrm{~T} / \mathrm{T})$ at $I L 2 R A$ were associated with an elevated risk of recurrence after BCG immunotherapy $[6,16]$. A germline deletion in the promoter area of $N F-k B$ was associated with an elevated risk of recurrence after BCG immunotherapy [17].

Furthermore, one study reported a predictive score that conferred likelihood of responding to BCG treatment. In the study, BCG response-associated SNPs, together with gender, number of tumor, and treatment scheme, were included in the risk model. Patients were classified into risk groups based on risk score: patients with low risk had a $90 \%$ likelihood of successful treatment (no recurrence), while patients with high risk had only a $25 \%$ likelihood of success (75\% recurrence) after BCG treatment. The AUC for this risk model was 0.82 [6]. This genetic risk classifier may be applied to personalized cancer care for patients before BCG treatment.

3.1.2. Glutathione (GSH) Pathway Genes. GSH pathway is involved in cell detoxification and antioxidation and may be associated with oncogenesis, prognosis, and response to treatment $[18,19]$. Therefore, genetic variants in the GSH pathway would influence cancer recurrence and response to BCG treatment after TURBT in NMIBC patients. Genetic variants in the GSH pathway may influence BCG response for patients after TURBT (Table 2).

A prospective study indicated that $h G P X 1(198 \mathrm{C} / \mathrm{T})$ was associated with risk of recurrence of NMIBC during
BCG immunotherapy [20]. Similar results were reported by Chiong et al. [21]. In several studies [22, 23], the polymorphisms of GSH pathway genes, for example, GSS(rs7265992), GSS(rs6060124), GSS(rs4911455), GPX2(rs10133290), GSTM4(rs560018), and GSTT1-positive, were significantly associated with increased risk of recurrence after BCG treatment. The polymorphisms of GPX5(rs377514) and GSTM3(rs4970737) had protective effects against recurrence after intravesical instillation BCG.

3.1.3. Nucleotide Excision Repair (NER) Gene. NER is one of the major DNA repair pathways related to the removal of a wide variety of DNA lesions, and some studies have revealed that the polymorphisms of DNA repair genes might be risk factors for many tumors [24]. A possible mechanism is that SNPs in NER genes may alter the DNA repair process and affect clinical outcome of bladder cancer.

The studies showed that patients who carried SNPs or mutations, such as rs1800975(A/A, in XPA) and Met1097Val (A/A, ERCC6), would have longer RFS during BCG treatment [25], while carrying ERCC2(312 A/A) and Lys939Gln(C/C, $X P C)$ was associated with high risk of recurrence and shorter RFS in BCG-treated patients [26, 27].

3.1.4. Pathways Genes of Apoptosis and Proliferation. The FAS/FASL system plays an important role in cell apoptosis, proliferation, and immune system regulation. Deregulation of the FAS/FASL system may lead to immune escape of tumor cells, thereby influencing BCG immunotherapy outcomes [28]. Lima et al. demonstrated that carriers of the FASL-844 $\mathrm{C} / \mathrm{C}$ genotype presented an increased risk of BCG treatment 
TABLE 2: Glutathione (GSH) pathway genes.

\begin{tabular}{|c|c|c|c|}
\hline Study & Sample (number) & Genes & Description \\
\hline Chiong et al. [21] & 99 & $h G P X 1(198 \mathrm{C} / \mathrm{T})$ & It was associated with an increased recurrence risk $(\mathrm{HR}=3.0 ; p=0.03)$ \\
\hline Kang et al. [23] & 135 & GSTT1-positive & It had a higher risk of BCG nonresponsiveness $(\mathrm{HR}=14 ; p=0.022)$ \\
\hline Ke et al. [22] & 191 & $\begin{array}{c}\text { GSS }(r s 7265992 \mathrm{~A} / \mathrm{A}) \\
\text { GSS }(r s 6060124 \mathrm{~A} / \mathrm{A}) \\
\text { GSS }(r s 4911455 \mathrm{C} / \mathrm{C}) \\
\text { GPX2(rs10133290 C/C) } \\
\text { GSTM4(rs560018 G/G) }\end{array}$ & They were associated with an elevated risk of recurrence \\
\hline Ke et al. [22] & 191 & $\begin{array}{c}\text { GPX5 }(r s 377514 \mathrm{C} / C) \\
\text { GSTM3(rs4970737 G/G) }\end{array}$ & They demonstrated protective association \\
\hline
\end{tabular}

failure and a decreased RFS after BCG treatment when compared with FASL-844 T allele carriers (HR: 1.92, $p=0.03$ ) [29]. This result was able to be validated in an independent study (HR: 1.71, $p=0.04$ ) [6].

Survivin, the expression product of the Survivin gene, is a member of the novel inhibitor of apoptosis protein (IAP) family, which is expressed in human malignancy, but almost undetectable in normal or well-differentiated adult tissues [30]. A study in patients undergoing BCG therapy showed that rs9904341(C/C) at Survivin was significantly correlated (HR: $0.35, p=0.009$ ) with a reduced risk of bladder cancer recurrence and a better RFS. Therefore, variant genotype CC also showed a protective association with bladder cancer [31].

3.1.5. Sonic Hedgehog (Shh) Pathway. Shh pathway is one of the major signal pathways that regulates cancer stem cells, cell proliferation, and differentiation, which is normally inactivated in adult tissues [32]. The activation of the Shh pathway has been found in bladder and many other tumors [33]. A study reported the correlation between BCG response and variations of genes in the Shh pathway. In this study, a total of 177 SNPs from 11 Shh pathway genes, including GLI1, GLI2, GLI3, GLI4, HHIP, STK36, SUFU, SHH, SMO, PTCH, and $P$ TCH2, were selected for genotyping. The results showed that rs6463089(A/A) and rs3801192(A/A) at GLI3 and GLI3 were significantly associated with increased risks of BCG failure $\left(p=2 \times 10^{-4}\right.$ and $9 \times 10^{-4}$, resp.) [34].

3.1.6. Other Genes. The natural resistance-associated macrophage protein 1 (NRAMP1) gene is the human counterpart of the murine $B c g$ gene and has been implicated in response to BCG in murine models [35]. Studies on NRAMP1 polymorphisms showed the same results, in which the NRAMP1 mutation $(\mathrm{D} 543 \mathrm{~N})$ was associated with an increased recurrence risk and a shorter RFS in patients treated with intravesical BCG therapy $[21,36]$.

Another study indicated that patients carrying the G/G genotype of rs5398 at intercellular adhesion molecule 1 (ICAM-1) presented a 2-fold increased risk of recurrence after BCG treatment $(p=0.032)$ and had a shorter RFS (80 versus 116 months) [6].

3.1.7. Copy Number Variations (CNVs). To date, many studies have been conducted to evaluate the correlation between
CNVs and bladder cancer in order to predict inherited risk and outcomes of disease to guide clinical decision making [37]. However, few articles were reported evaluating the relationship between CNVs and BCG response. To our knowledge, only one study has been reported and evaluated the role of loss of heterozygosity (LOH) in the IFN- $a$ (chromosome $9 \mathrm{p} 21)$ to predict BCG response in patients who underwent TURBT. Results showed that LOH at the IFN-a locus was an independent predictor of BCG response $(p=0.002)$ and was significantly associated with increased BCG failure $(p<$ $0.0001)$ [7].

3.2. Somatic Variation. Only one study, to our knowledge, has reported an association between somatic genetic alterations and BCG response in bladder cancer patients. The study demonstrated the effectiveness of an E2F4 signature (somatic mutations and gene expression) for predicting BCG response in patients with bladder cancer [38]. E2F4 is a member of the E2F transcription factor family, which plays critical roles in cell-cycle progression and differentiation [39]. Abnormal expression of E2F4 or mutation of E2F4 targeted genes caused the malfunction of cell-cycle controls and was associated with various tumors [40, 41].

In a study conducted by Cheng et al., an E2F4 score based on mutation and expression status of E2F4 was established for each patient. In patients with positive E2F4 scores (E2F4 $>0$ group), BCG immunotherapy could significantly improve the clinical outcomes of NMIBC after TURBT. However, BCG may not improve prognosis in patients with negative scores (E2F4 < 0 group) [38].

3.3. Epigenetic. DNA methylation is a common epigenetic change that can regulate the expression of genes, and it is closely correlated with carcinogenesis and disease progression. Many studies have indicated that DNA methylation testing could be applied to cancer detection, prognosis, and treatment response, including bladder cancer (BCa) $[42,43]$. Several studies investigated the relationships between gene methylation and disease outcomes in patients with NMIBC treated with BCG instillation.

In a study conducted by Agundez et al. [9], the roles of the methylation of 25 tumor suppressor genes (TSG) were evaluated, and results showed that the methylation status of TSGs (STK11, MSH6, BRCA1, PAX5A, MGMT, CDH13, etc.) 
was associated with the clinical outcome of patients with T1G3 tumors undergoing BCG treatment. Another study evaluated the role of polyamine-modulated factor-1 (PMF1) methylation in predicting BCG response and revealed that the methylation status of $P M F-1$ was associated with an increased recurrence $(p=0.026)$, progression $(p=0.01)$, and shorter disease-specific survival $(p=0.03)$ [8]. An independent study also reported that myopodin methylation was associated with an increased risk of recurrence $(p=0.011)$, progression ( $p=0.03$ ), and shorter disease-specific survival $(p=0.028)$ in patients treated with BCG therapy [10].

The results of these studies suggested a potential clinical application of gene methylation testing for predicting BCG response in patients with $\mathrm{BCa}$.

\section{Conclusion}

At present, BCG is considered to be the best adjuvant therapy after TURBT for preventing recurrence and delaying progression in NMIBC. However, many patients eventually recur or progress to BCG refractory bladder cancer. Thus, it is essential to be able to determine who will respond well to BCG treatment in order to provide individualized bladder cancer care. Recently, genetic variations in molecules involved in the BCG mechanism of action have also been considered good candidates for predicting treatment outcome.

Although studies based on genetic variants showed promising results, most lacked validation. Nevertheless, genetic variations would be useful predictors for BCG treatment outcome. Their clinical applications should be further evaluated in large-scale validation studies. In addition, other biomarkers based on different "-omics" technologies should also be considered in future studies. A single predictive biomarker seems insufficient due to the fact that BCG response is a multistep process, so it would be meaningful to explore the predictive value of more combinations of biomarkers. Therefore, it would be of use to develop a panel or profile based on high-risk alleles or genes to predict BCG response and guide clinical intravesical treatment decisions.

\section{Competing Interests}

The authors declare that there is no conflict of interests regarding the publication of this paper.

\section{Authors' Contributions}

Ning Zhang and Guangliang Jiang are equal contributors and co-first authors.

\section{Acknowledgments}

This study was partially funded by the National Natural Science Foundation of China (Grant no. 81570607) to Xiang Wang and by the National Natural Science Foundation of China (Grant no. 81402339) to Rong Na.

\section{References}

[1] R. L. Siegel, K. D. Miller, and A. Jemal, “Cancer statistics, 2016," CA Cancer Journal for Clinicians, vol. 66, no. 1, pp. 7-30, 2016.

[2] M. Babjuk, A. Bohle, M. Burger et al., "EAU guidelines on nonmuscle-invasive urothelial carcinoma of the bladder: update 2016," European Urology, In press.

[3] J. A. Witjes, E. Compérat, N. C. Cowan et al., "EAU guidelines on muscle-invasive and metastatic bladder cancer: summary of the 2013 guidelines," European Urology, vol. 65, no. 4, pp. 778792, 2014.

[4] Y. Miyata and H. Sakai, "Predictive markers for the recurrence of nonmuscle invasive bladder cancer treated with intravesical therapy," Disease Markers, vol. 2015, Article ID 857416, 10 pages, 2015.

[5] C. Lenormand, J. Couteau, F. X. Nouhaud et al., "Predictive value of NRAMP1 and HGPX1 gene polymorphism for maintenance BCG response in non-muscle-invasive bladder cancer," Anticancer Research, vol. 36, no. 4, pp. 1737-1743, 2016.

[6] L. Lima, D. Oliveira, J. A. Ferreira et al., "The role of functional polymorphisms in immune response genes as biomarkers of bacille Calmette-Guérin (BCG) immunotherapy outcome in bladder cancer: establishment of a predictive profile in a Southern Europe population," BJU International, vol. 116, no. 5, pp. 753-763, 2016.

[7] T. Cai, G. Nesi, M. Dal Canto et al., "Loss of heterozygosis on IFN- $\alpha$ locus is a prognostic indicator of bacillus CalmetteGuerin response for nonmuscle invasive bladder cancer," Journal of Urology, vol. 183, no. 5, pp. 1738-1743, 2010.

[8] M. Alvarez-Múgica, J. M. Fernández-Gómez, V. Cebrian, F. Fresno, S. Escaf, and M. Sánchez-Carbayo, "Polyaminemodulated factor-1 methylation predicts bacillus CalmetteGuérin response in patients with high-grade non-muscleinvasive bladder carcinoma," European Urology, vol. 63, no. 2, pp. 364-370, 2013.

[9] M. Agundez, L. Grau, J. Palou, F. Algaba, H. Villavicencio, and M. Sanchez-Carbayo, "Evaluation of the methylation status of tumour suppressor genes for predicting bacillus CalmetteGuérin response in patients with T1G3 high-risk bladder tumours," European Urology, vol. 60, no. 1, pp. 131-140, 2011.

[10] M. Alvarez-Múgica, V. Cebrian, J. M. Fernández-Gómez, F. Fresno, S. Escaf, and M. Sánchez-Carbayo, "Myopodin methylation is associated with clinical outcome in patients with T1G3 bladder cancer," Journal of Urology, vol. 184, no. 4, pp. 1507-1513, 2010.

[11] T. C. M. Zuiverloon, A. J. M. Nieuweboer, H. Vékony, W. J. Kirkels, C. H. Bangma, and E. C. Zwarthoff, "Markers predicting response to bacillus Calmette-Guérin immunotherapy in high-risk bladder cancer patients: a systematic review," European Urology, vol. 61, no. 1, pp. 128-145, 2012.

[12] G. Redelman-Sidi, M. S. Glickman, and B. H. Bochner, "The mechanism of action of BCG therapy for bladder cancer-a current perspective," Nature Reviews Urology, vol. 11, no. 3, pp. 153-162, 2014.

[13] D. Leibovici, H. B. Grossman, C. P. Dinney et al., "Polymorphisms in inflammation genes and bladder cancer: from initiation to recurrence, progression, and survival," Journal of Clinical Oncology, vol. 23, no. 24, pp. 5746-5756, 2005.

[14] D. Ahirwar, P. Kesarwani, P. K. Manchanda, A. Mandhani, and R. D. Mittal, "Anti- and proinflammatory cytokine gene 
polymorphism and genetic predisposition: association with smoking, tumor stage and grade, and bacillus Calmette-Guérin immunotherapy in bladder cancer," Cancer Genetics and Cytogenetics, vol. 184, no. 1, pp. 1-8, 2008.

[15] D. K. Ahirwar, A. Mandhani, A. Dharaskar, P. Kesarwani, and R. D. Mittal, "Association of tumour necrosis factor- $\alpha$ gene (T-1031C, C-863A, and C-857T) polymorphisms with bladder cancer susceptibility and outcome after bacille Calmette-Guérin immunotherapy," BJU International, vol. 104, no. 6, pp. 867-873, 2009.

[16] D. K. Ahirwar, A. Agrahari, A. Mandhani, and R. D. Mittal, "Cytokine gene polymorphisms are associated with risk of urinary bladder cancer and recurrence after BCG immunotherapy," Biomarkers, vol. 14, no. 4, pp. 213-218, 2009.

[17] D. K. Ahirwar, A. Mandhani, and R. D. Mittal, "IL-8 -251 T > A Polymorphism is associated with bladder cancer susceptibility and outcome after BCG immunotherapy in a northern Indian cohort," Archives of Medical Research, vol. 41, no. 2, pp. 97-103, 2010.

[18] A. Savic-Radojevic, J. Mimic-Oka, M. Pljesa-Ercegovac et al., "Glutathione S-transferase-P1 expression correlates with increased antioxidant capacity in transitional cell carcinoma of the urinary bladder," European Urology, vol. 52, no. 2, pp. 470477, 2007.

[19] E. Drozd, J. Krzysztoń-Russjan, J. Marczewska et al., "Upregulation of glutathione-related genes, enzyme activities and transport proteins in human cervical cancer cells treated with doxorubicin," Biomedicine \& Pharmacotherapy, vol. 83, pp. 397406, 2016.

[20] H. Zhao, D. Liang, H. B. Grossman, and X. Wu, "Glutathione peroxidase 1 gene polymorphism and risk of recurrence in patients with superficial bladder cancer," Urology, vol. 66, no. 4, pp. 769-774, 2005.

[21] E. Chiong, A. Kesavan, R. Mahendran et al., "NRAMP1 and hGPX1 gene polymorphism and response to bacillus CalmetteGuérin therapy for bladder cancer," European Urology, vol. 59, no. 3, pp. 430-437, 2011.

[22] H.-L. Ke, J. Lin, Y. Ye et al., "Genetic variations in glutathione pathway genes predict cancer recurrence in patients treated with transurethral resection and bacillus calmette-guerin instillation for non-muscle invasive bladder cancer," Annals of Surgical Oncology, vol. 22, no. 12, pp. 4104-4110, 2015.

[23] H. W. Kang, D.-U. Tchey, C. Yan et al., “The predictive value of GSTT1 polymorphisms in predicting the early response to induction BCG therapy in patients with non-muscle invasive bladder cancer," Urologic Oncology: Seminars and Original Investigations, vol. 32, no. 4, pp. 458-465, 2014.

[24] E. L. Goode, C. M. Ulrich, and J. D. Potter, "Polymorphisms in DNA repair genes and associations with cancer risk," Cancer Epidemiology Biomarkers and Prevention, vol. 11, no. 12, pp. 1513-1530, 2002.

[25] J. Gu, H. Zhao, C. P. Dinney et al., "Nucleotide excision repair gene polymorphisms and recurrence after treatment for superficial bladder cancer," Clinical Cancer Research, vol. 11, no. 4, pp. 1408-1415, 2005.

[26] R. Gangwar, A. Mandhani, and R. D. Mittal, “XPC gene variants: a risk factor for recurrence of urothelial bladder carcinoma in patients on BCG immunotherapy," Journal of Cancer Research and Clinical Oncology, vol. 136, no. 5, pp. 779-786, 2010.

[27] R. Gangawar, D. Ahirwar, A. Mandhani, and R. D. Mittal, "Impact of nucleotide excision repair ERCC2 and base excision repair APEX1 genes polymorphism and its association with recurrence after adjuvant BCG immunotherapy in bladder cancer patients of North India," Medical Oncology, vol. 27, no. 2, pp. 159-166, 2010.

[28] M. Ehrenschwender and H. Wajant, "The role of FasL and Fas in health and disease," Advances in Experimental Medicine and Biology, vol. 647, pp. 64-93, 2009.

[29] L. Lima, J. A. Ferreira, A. Tavares et al., "FASL polymorphism is associated with response to bacillus Calmette-Guérin immunotherapy in bladder cancer," Urologic Oncology, vol. 32, no. 1, pp. 44.el-44.e7, 2014.

[30] D. C. Altieri, "Survivin, cancer networks and pathway-directed drug discovery," Nature Reviews Cancer, vol. 8, no. 1, pp. 61-70, 2008.

[31] P. K. Jaiswal, A. Goel, A. Mandhani, and R. D. Mittal, "Functional polymorphisms in promoter survivin gene and its association with susceptibility to bladder cancer in north indian cohort," Molecular Biology Reports, vol. 39, no. 5, pp. 5615-5621, 2012.

[32] S. J. Scales and F. J. de Sauvage, "Mechanisms of Hedgehog pathway activation in cancer and implications for therapy," Trends in Pharmacological Sciences, vol. 30, no. 6, pp. 303-312, 2009.

[33] L. Yang, G. Xie, Q. Fan, and J. Xie, "Activation of the hedgehogsignaling pathway in human cancer and the clinical implications," Oncogene, vol. 29, no. 4, pp. 469-481, 2010.

[34] M. Chen, M. A. T. Hildebrandt, J. Clague et al., "Genetic variations in the sonic hedgehog pathway affect clinical outcomes in non-muscle-invasive bladder cancer," Cancer Prevention Research, vol. 3, no. 10, pp. 1235-1245, 2010.

[35] S. A. Kadhim, J. L. Chin, E. Batislam, S. J. Karlik, B. Garcia, and E. Skamene, "Genetically regulated response to intravesical Bacillus Calmette Guerin immunotherapy of orthotopic murine bladder tumor," The Journal of Urology, vol. 158, no. 2, pp. 646652, 1997.

[36] M. Decobert, H. LaRue, A. Bergeron et al., "Polymorphisms of the human NRAMP1 gene are associated with response to bacillus Calmette-Guerin immunotherapy for superficial bladder cancer," The Journal of Urology, vol. 175, no. 4, pp. 15061511, 2006.

[37] M. S. Nørskov, R. Frikke-Schmidt, S. E. Bojesen, B. G. Nordestgaard, S. Loft, and A. Tybjærg-Hansen, "Copy number variation in glutathione-S-transferase T1 and M1 predicts incidence and 5year survival from prostate and bladder cancer, and incidence of corpus uteri cancer in the general population," Pharmacogenomics Journal, vol. 11, no. 4, pp. 292-299, 2011.

[38] C. Cheng, F. S. Varn, and C. J. Marsit, "E2F4 program is predictive of progression and intravesical immunotherapy efficacy in bladder cancer," Molecular Cancer Research, vol. 13, no. 9, pp. 1316-1324, 2015.

[39] B. D. Rowland and R. Bernards, "Re-evaluating cell-cycle regulation by E2Fs," Cell, vol. 127, no. 5, pp. 871-874, 2006.

[40] S. Schwemmle and G. P. Pfeifer, "Genomic structure and mutation screening of tile E2F4 gene in human tumors," International Journal of Cancer, vol. 86, no. 5, pp. 672-677, 2000.

[41] R. F. Souza, J. Yin, K. N. Smolinski et al., "Frequent mutation of the E2F-4 cell cycle gene in primary human gastrointestinal tumors," Cancer Research, vol. 57, no. 12, pp. 2350-2353, 1997. 
[42] E. N. Gal-Yam, Y. Saito, G. Egger, and P. A. Jones, "Cancer epigenetics: modifications, screening, and therapy," Annual Review of Medicine, vol. 59, pp. 267-280, 2008.

[43] M. B. Scher, M. B. Elbaum, Y. Mogilevkin et al., "Detecting DNA methylation of the BCL2, CDKN2A and NID2 genes in urine using a nested methylation specific polymerase chain reaction assay to predict bladder cancer," Journal of Urology, vol. 188, no. 6, pp. 2101-2107, 2012. 


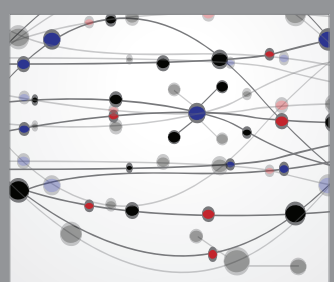

The Scientific World Journal


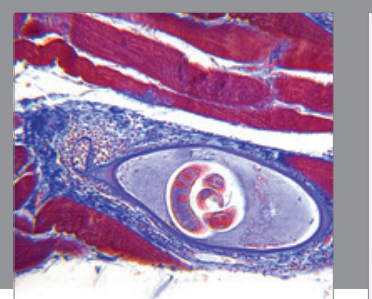

Gastroenterology Research and Practice

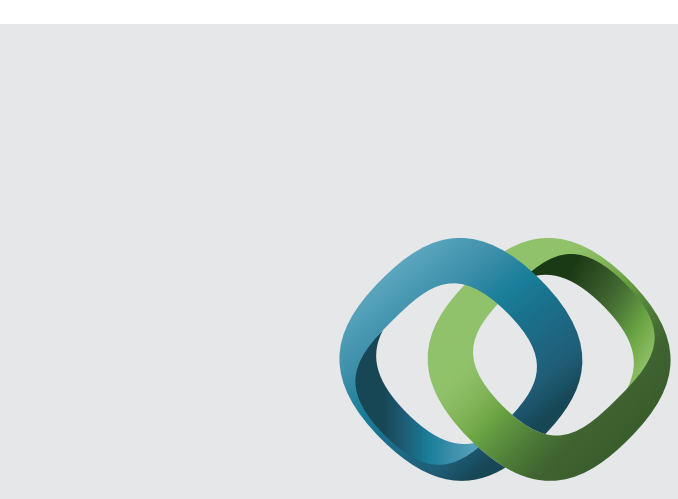

\section{Hindawi}

Submit your manuscripts at

http://www.hindawi.com
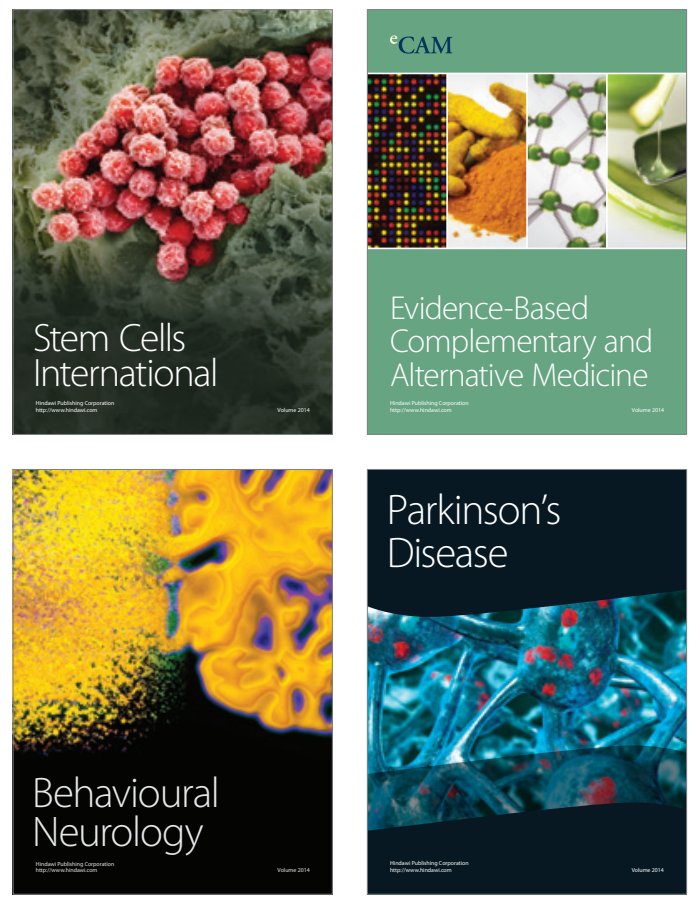
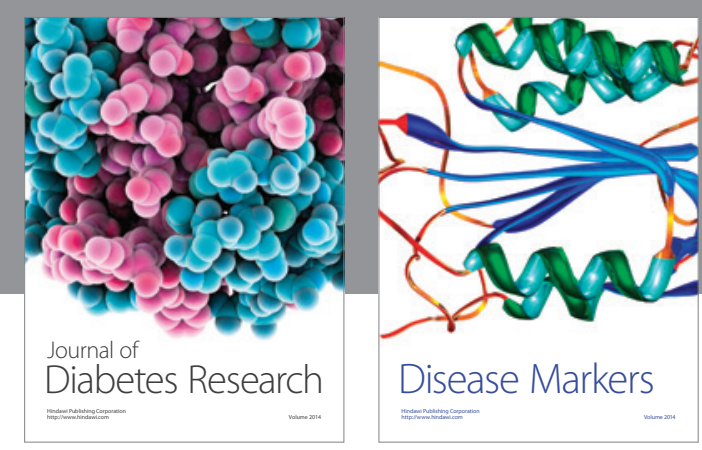

Disease Markers
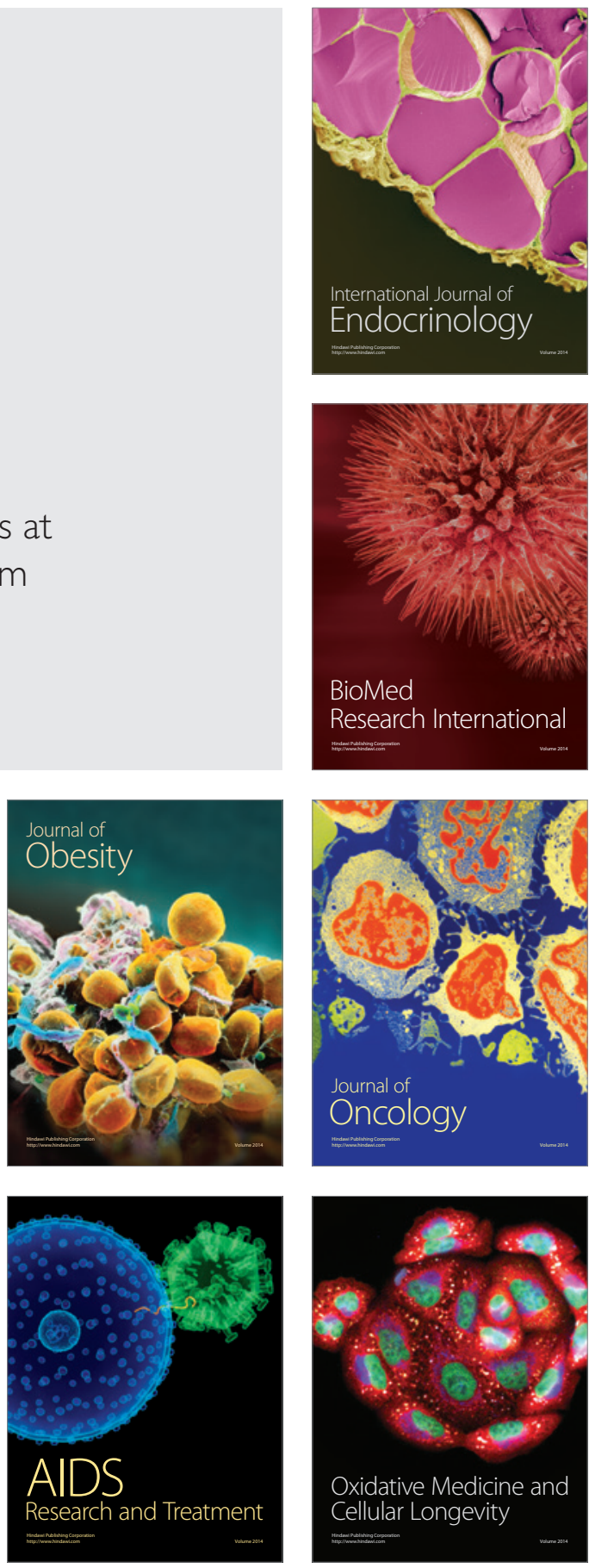